\title{
Utilization of lyophilized platelet-rich plasma in esthetic facial treatment
}

\author{
Teh-Yang Cheng, MD iD \\ Department of Dermatology, National Taiwan University Hospital, Taipei City, Taiwan
}

\begin{abstract}
Platelet-rich plasma (PRP) has a variety of functions. Activated platelets release various growth factors and are involved in the tissue repair process. Growth factors from platelets induce fibroblast activation, which produces a new extracellular matrix and collagen. The PRP treatment has been widely used in many surgical and medical fields. However, uncertainties in the quality of the PRP preparation may cause unpredictability in the treatment results. The standardized separation method with quantization of lyophilized platelet-rich plasma (L-PRP) might enhance treatment accuracy. After processing in central laboratory, L-PRP is packed in vials in powder form and readily used immediately after reconstitution. We utilized clinical photography, Visia, DermaLab USB, and histology examination to compare the results before and after treatments. Besides improvements in clinical photos and Visia scores, ultrasound images showed obvious increasing in collagen densities in dermal and subcutaneous layers. The histology study suggested increased collagen formation but without signs of fibrotic change or foreign body reaction. The elastin stain suggested an increased elastic fiber density. The improvement noted by the instruments and histology findings correlated with the improvement of skin texture, decreased wrinkles, and positive clinical outcomes. These results highlight L-PRP's potential as an efficient and convenient process compared to the original PRP in skin rejuvenation treatment.
\end{abstract}

Keywords: Iyophilized platelet-rich plasma; neo-collagenesis; platelet-rich plasma; skin rejuvenation

\section{Introduction}

Platelets play a crucial role in hemostasis and wound healing. Various growth factors have been identified within the alpha granules [1]. Platelet-rich plasma (PRP) is obtained by isolation of centrifuged autologous blood. Therefore, PRP devices can traditionally be categorized into lower (2.5-3 times baseline concentration) and higher (5-9 times baseline concentration) concentration systems [2]. However, growth factor levels are not routinely measured in clinical practice.

Lyophilized platelet-rich plasma (L-PRP) is a technology that can further preserve purified PRP for a prolonged period. After collecting autologous blood into blood bags containing citrate phosphate dextrose adenine-1 (CPDA-1), they are sent to the central laboratory for centrifugation and further processing. The L-PRP is disinfected and sent back to the medical facility with platelet-derived growth factor-BB (PDGF-BB) levels labeled ng per vial. L-PRP can be easily reconstituted with mixtures of normal saline and lidocaine before each treatment session.

PRP has been widely used in various fields of medical treatment, including hastening tendon repair, reconstructive medicine, wound healing, hair loss, and esthetic medicine. Many studies have shown improvement in skin texture, wrinkles, and facial volume [3,4].

Received August 19, 2020; Revised September 21, 2020; Accepted September 21, 2020

Corresponding author: Teh-Yang Cheng

E-mail: jerome.cheng@gmail.com

Teh-Yang Cheng's current affiliation: Infinite Delight Aesthetic Clinic, Taipei City, Taiwan

This is an Open Access article distributed under the terms of the Creative Commons Attribution Non-Commercial License (http://creativecommons.org/licenses/by-nc/4.0), which permits unrestricted non-commercial use, distribution, and reproduction in any medium, provided the original work is properly cited.

Copyright (c) 2020 Korean Society of Korean Cosmetic Surgery and Medicine (KSKCS \& KCCS). 
We report 3 cases of L-PRP usage in facial esthetic treatment. In addition to clinical photo comparisons, we measured the change in collagen density after facial treatment. Besides, clinical photo comparison was used for an additional patient receiving treatment for striae distensae.

\section{Case report}

\section{Study design and subjects}

Patients gave their formal consents for the use of their personal and medical information as well as photos and laboratory data in the publication of this case report.

\section{Lyophilized platelet-rich plasma preparation}

The preparation of L-PRP was performed in the central laboratory of Spirit Scientific Co., Ltd. Taiwan Branch (Taipei, Taiwan). For each patient, $250 \mathrm{ml}$ of autologous blood was collected at once into a blood bag containing CPDA-1. Upon receiving the blood bag, a series of separations and concentrations of platelets was performed. The platelets were dispensed into vials $\left(1 \times 10^{9}\right.$ per vial). They were partially activated before the vacuum freeze-drying process. After gamma-ray sterilization of the freeze-dried PRP, PDGF-BB was measured and labeled before being sent to the original medical facility. Using a single procedure, 20 vials of lyophilized powder were produced. Under a scanning electron microscope, most of the platelets retained their original shapes (Fig. 1). The lyophilized powder was stored at $25 \pm 2$ degrees Celsius and at 1 atmospheric pressure. In addition, different centrifugation systems for producing PRP have been developed with different leukocyte concentrations. According to the literature, the reference ranges of leukocyte concentration are from 0 to $50 \times 10^{3} / \mu \mathrm{l}$ [5]. In this system, the range of leukocyte concentrations was approximately $0.1-0.3 \times 10^{3} / \mu$ l.

\section{Lyophilized platelet-rich plasma application}

Before the treatment session, each vial of L-PRP was reconstituted in $1 \mathrm{ml}$ of normal saline (with or without mixing with $2 \%$ lidocaine) using a sterile syringe. The platelet-rich solution was administered intradermally, subcutaneously, and supraperiosteally. Approximately 4 to 7 vials were administered for each treatment session. Thus, 20 L-PRP vials can be divided into several sessions. Each session was 2 to 4 weeks apart.

\section{VISIA}

VISIA is an imaging analytic product produced by Canfield Scientific, Inc. (Canfield Imaging Systems, Parsippany, NJ, USA). It is widely used in the noninvasive measurement of facial skin wrinkles, pores, and texture in the dermatology field. We utilized VISIA for patients before the first and second treatments, and at the follow-up clinic ( 1 month after the second treatment). Makeup was removed before clinical imaging. Comparison data were acquired after the follow-up session.

\section{DermaLab USB}

DermaLab USB (Cortex Technology, Hadsund, Denmark) is a parameter-ultrasound skin analysis instrument. It was produced by Cortex Technology. We measured the patients' skin using a $20 \mathrm{MHz} 2$ dimensional-scanning probe. The intensity of collagen was displayed using a color scale. Yellow indicates the highest reflection, while green indicates the lowest reflection.

\section{Histology}

We obtained consent for skin punch biopsy ( $3 \mathrm{~mm}$ ) before treatment and at the follow-up clinic (one month after the second treatment) from the second and third patients. Biopsies were performed in the left preauricular area. The biopsied specimens were fixed in formalin, embedded in paraffin, cut at $3 \mu \mathrm{m}$, and stained with Trichrome-Masson and Miller elastic stain according to standard procedures.
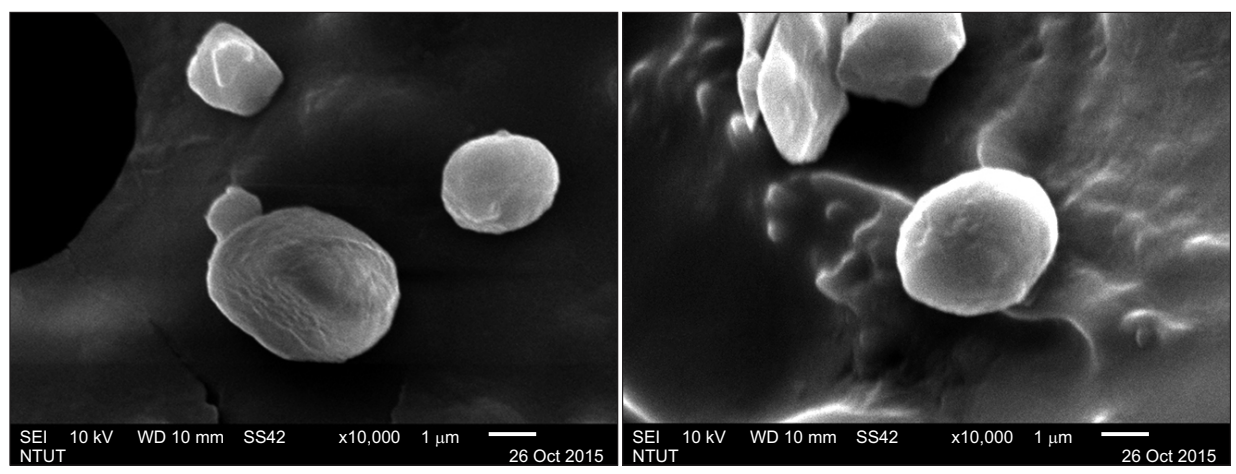

Fig. 1. After reconstitution of the lyophilized platelet rich plasma, most of the platelets retained their original shapes (scanning electron microscope, 10,000×). 


\section{Case I}

This is a 35-year-old female who presented with a sunken temple, sunken cheeks, and coarse skin texture. The PDGF-BB level was $18.5 \mathrm{ng} /$ vial for her L-PRP. The L-PRP solution was injected into her temporal areas, forehead, and cheeks intradermally, subcutaneously, and supraperiosteally. She received 4 treatment sessions (1-month-apart), but the cheeks were treated only during the last 2 sessions. Only minor bruising was noted after the treatments. After comparing clinical photos, we noted that the outline of her forehead, temporal area, and cheeks became smooth (Fig. 2A, B). Few concavities were noted on her face; however, the wrinkles and pores on her skin texture, measured by VISIA showed apparent improvements (Fig. 2C).

\section{Case II}

This is a 30-year-old female with coarse skin texture and loosened periorbital skin. The PDGF-BB level was $18.1 \mathrm{ng} / \mathrm{vial}$ for her L-PRP. She received 2 treatment sessions a month apart. VISIA and DermaLab USB measurements were performed before the first and second treatments and at the follow-up clinic (a month after the second treatment). Punch biopsies were performed before the first treatment and at the follow-up clinic. The fine lines and the texture on her lower eyelids improved noticeably (Fig. 3A-C). The ultrasound images obtained by DermaLab USB on the lower eyelid suggested a remarkable increase in collagen density either within the dermal layer or subcutaneous layer (Fig. 3D). The histology study suggested increased collagen formation but without signs of fibrotic change or foreign body reaction. The elastin stain suggested an increased elastic fiber density (Fig. 3E, F). A short duration of minor itching was noted after the second session.

\section{Case III}

This is a 33-year-old female with fair skin conditions. The PDGF-BB level was $11.2 \mathrm{ng} / \mathrm{vial}$ for her L-PRP. The treatment protocol was the same as in Case II. Improvement in skin textures and outline were noted (Fig. 4A). We measured the collagen density in the supra-eyebrow area using DermaLab USB (Fig. 4B). The color scales of ultrasound images suggested increased collagen density in at the dermis and subcutaneous layers. The histology study suggested an increase in collagen and elastic fibers (Fig. 4C, D).

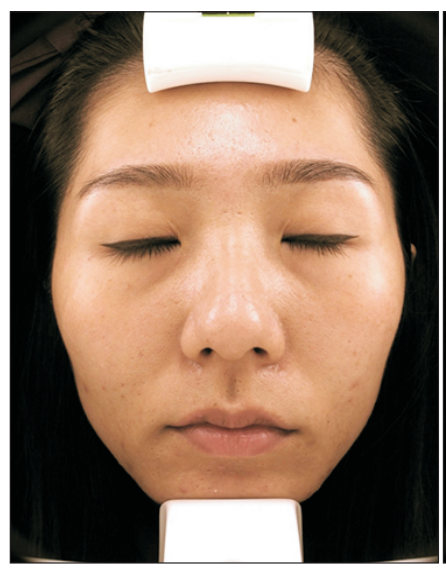

Before

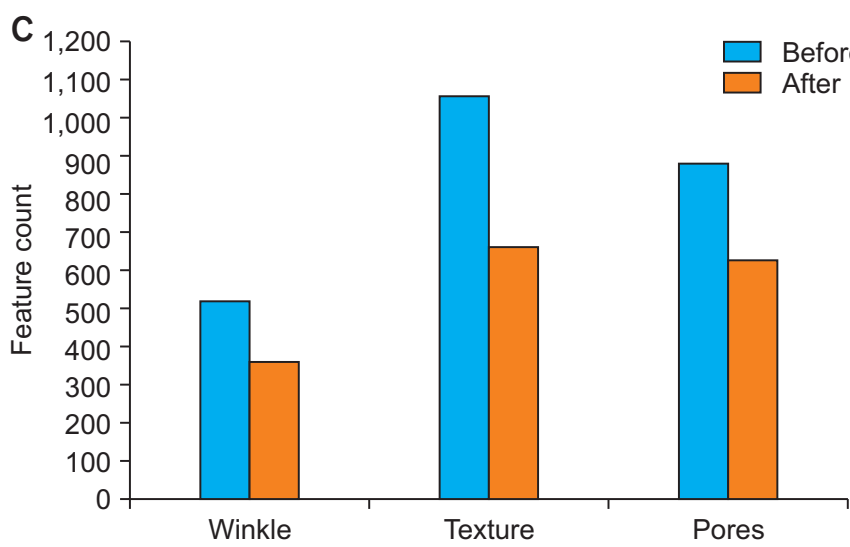

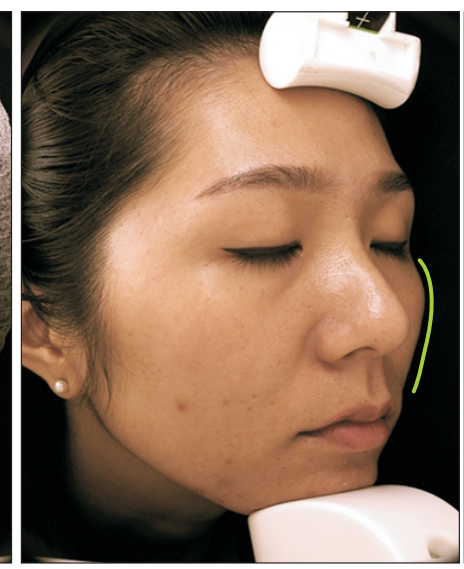

Before

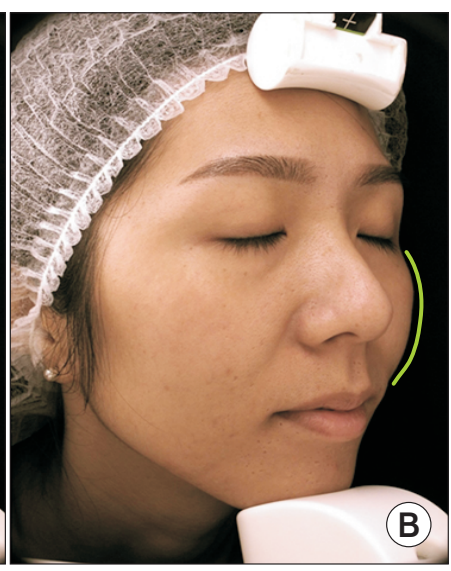

1 month after 4 th treatment

Fig. 2. (A, B) Four sessions of treatments (1-month-apart) shows improvement in the appearance of forehead, temporal, and cheeks in this 35-year-old female. (C) The skin texture, wrinkles, and pores were improved over baseline at post-treatment (average improvement rate: $32 \%$ ). 


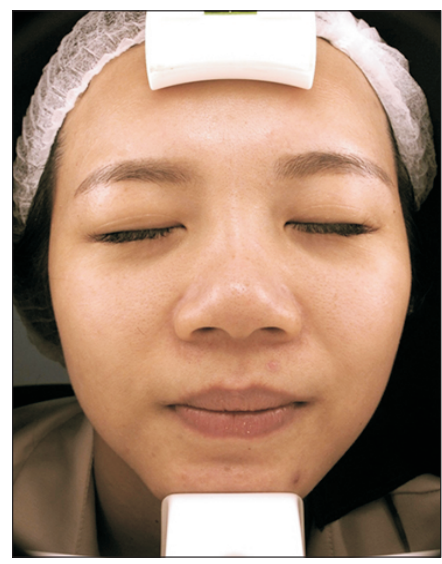

Before

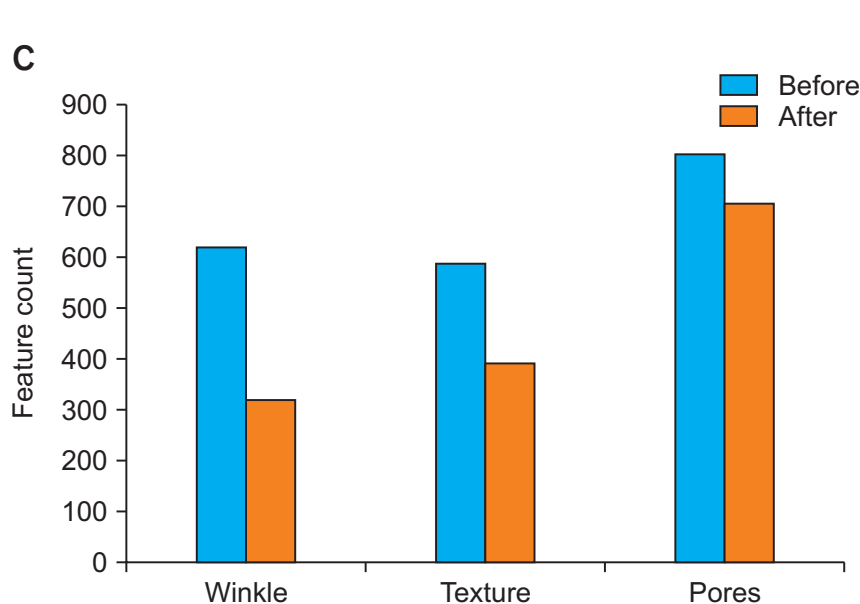

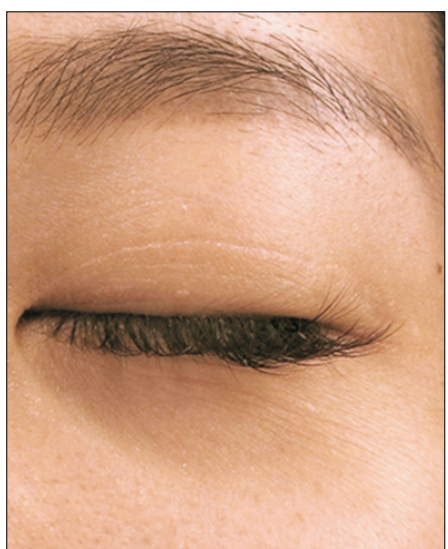

Before

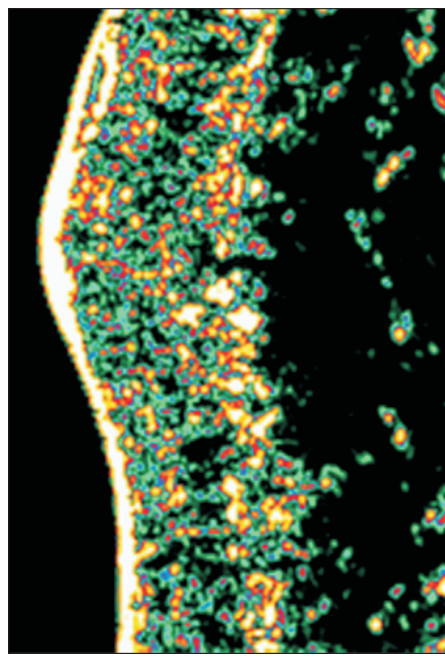

Before

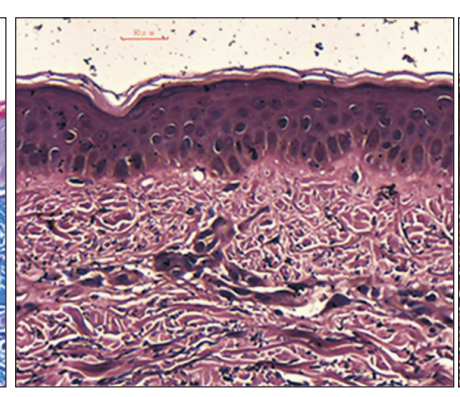

Before

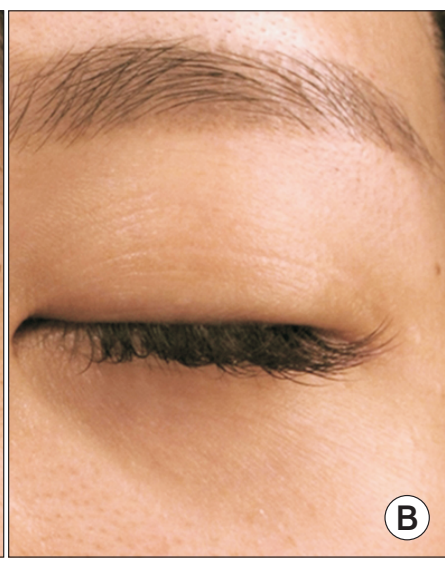

1 month after 4 th treatment

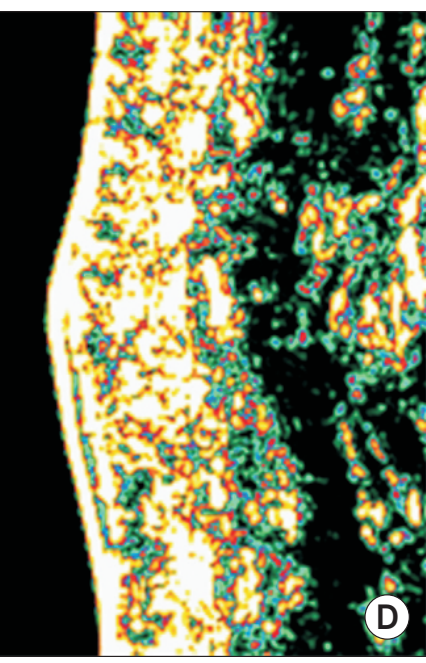

1 month after 2nd treatment

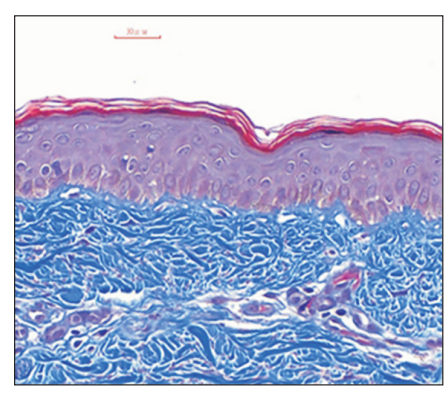

Before

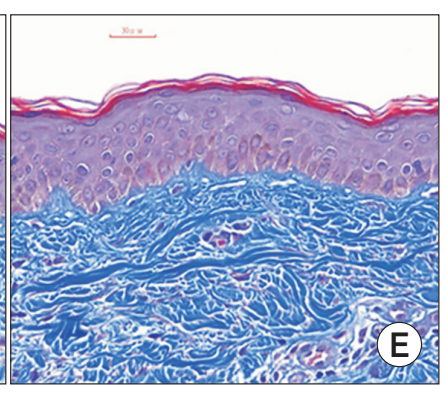

1 month after 2nd treatment

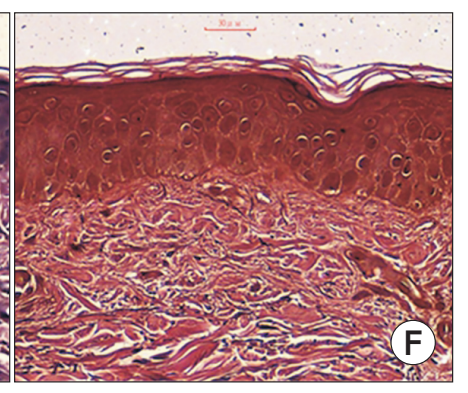

1 month after 2 nd treatment

Fig. 3. (A, B) Two sessions of treatments (1-month-apart) shows improvement in the facial skin and lower eyelid in this 30-year-old female.

(C) The skin texture, wrinkles and pores were improved over baseline at post-treatment (average improvement rate: $31 \%$ ). (D) The ultrasound images of eyelid collagen density before (left) and post-treatment (right). (E, F) Histology of eyelid collagen density before (left) and posttreatment (right) (E: Masson's Trichrome, 400×; F: Miller elastin stain, 400×).

\section{Case IV}

This is a 30-year-old female who complained of striae distensae on her calves. The PDGF-BB level was $15.6 \mathrm{ng} /$ vial for her L-PRP. The treatment protocol involved spraying the reconstituted L-PRP solution after micro-needling on her calves. One milliliter of the L-PRP solution was used for $25 \mathrm{~cm}^{2}$ of the skin.
She received 3 sessions of treatment 2 weeks apart. Eight vials of L-PRP were used for the first 2 sessions, and 6 vials for the 3 rd session. Clinical improvement was noted (Fig. 5). 


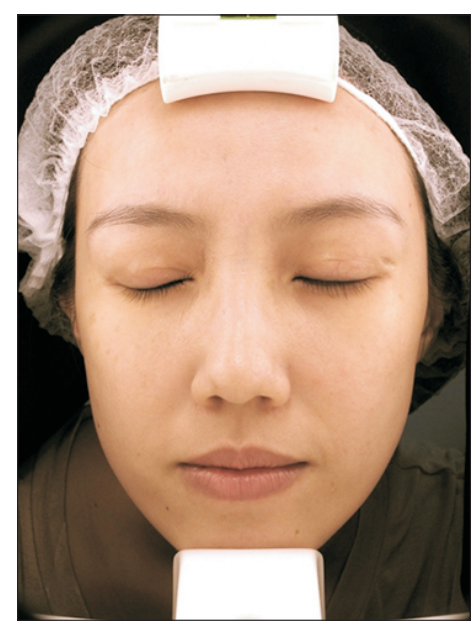

Before

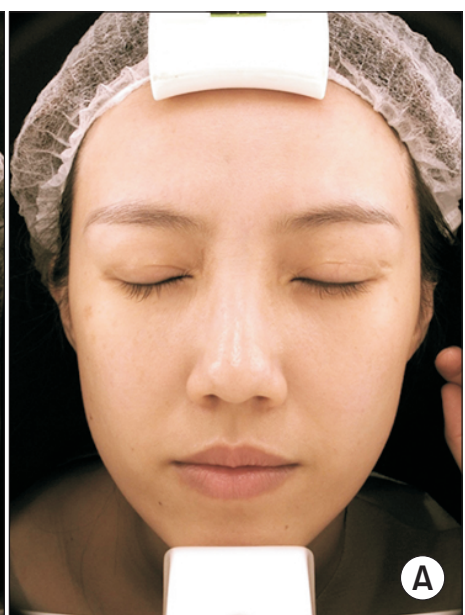

1 month after 4th treatment

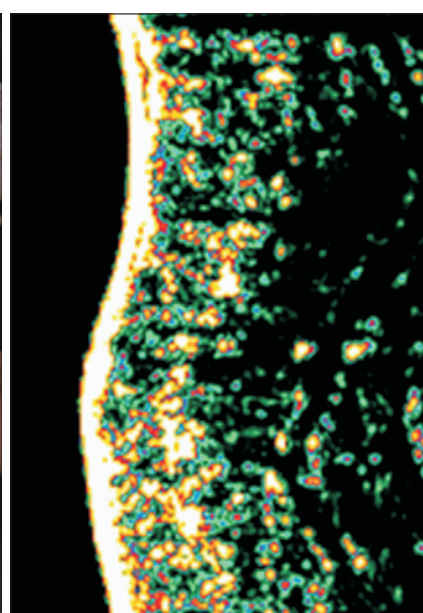

Before

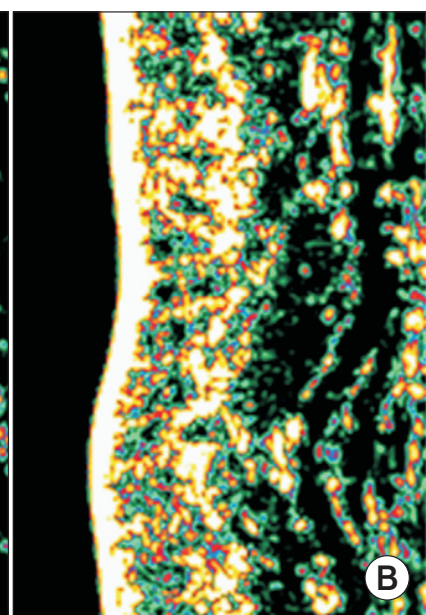

1 month after 2nd treatment

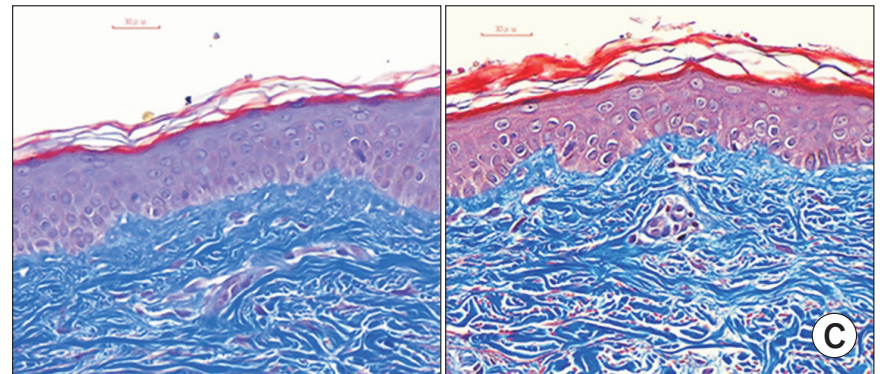

Before

1 month after 2 nd treatment

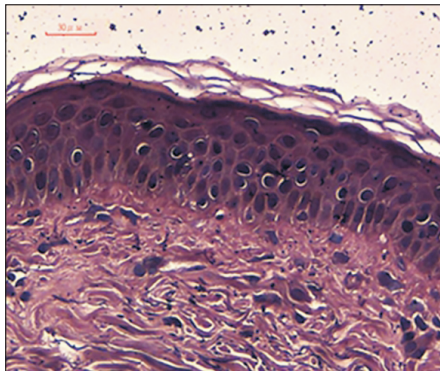

Before

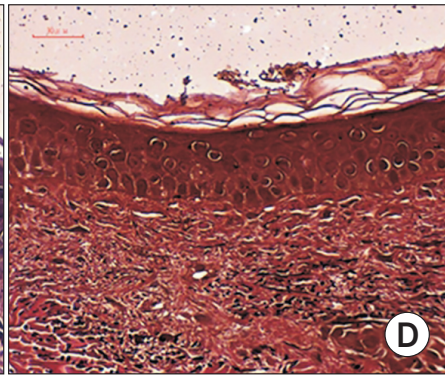

1 month after 2 nd treatment

Fig. 4. (A) Two sessions of treatments (1-month-apart) shows improvement in the facial skin texture and outline in this 33-year-old female. (B) The ultrasound images of supra-eyebrow collagen density before (left) and post-treatment (right). (C, D) Histology of supra-eyebrow collagen density before (left) and post-treatment (right) (C: Masson's Trichrome, 400×; D: Miller elastin stain, 400×).

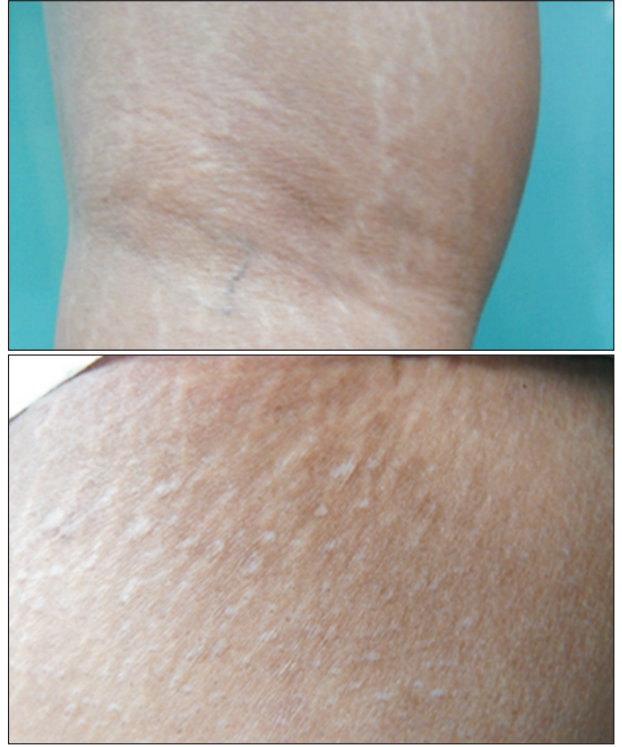

Before

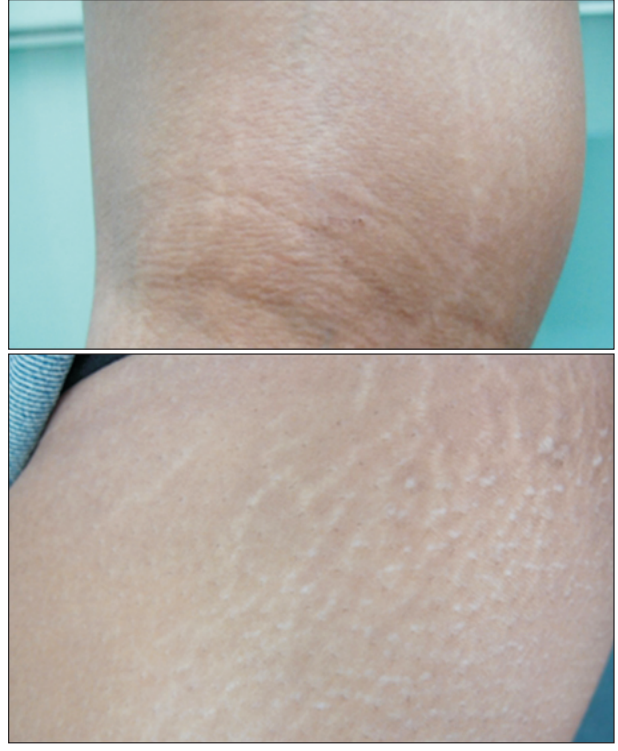

2 weeks after 3rd treatment
Fig. 5. Three sessions of treatments (2-week-apart) shows improvement of the striae distensae on patient's calves. 


\section{Discussion}

The use of PRP in medical treatments was considered relatively safe and efficient. There are no current standard guidelines for obtaining PRP to yield the most efficacious plasma solution or platelet concentration [3]. In addition, over-concentrated or large amounts of injection might yield poor treatment results. This might be related to the amount of white blood cells or proinflammatory substances within the plasma [5].

PRP may hasten tissue repair and is widely used in orthopedic procedures, reconstructive medicine, and wound healing promotion [6]. PRP has become more popular in facial esthetic treatment. In this series of cases receiving L-PRP treatments, clinical improvements and positive clinical feedback were noted. Decreasing fine lines (wrinkles), improvement of skin textures, and smoother facial outlines were observed. Increasing collagen density within the dermis, and subcutaneous layers were noted with the aid of an ultrasound imaging device (DermaLab USB). Tissue biopsy suggested that the new collagen was formed without fibrotic change or foreign body reaction. In addition, increased elastic fibers were observed. Therefore, the improvement in skin texture and appearance might be explained.

PRP contains several growth factors that might play a role in the activation of fibroblasts and tissue regeneration. Growth factors secreted from platelets are attributed to tissue repairs, such as platelet-derived growth factor (PDGF), vascular endothelial growth factor (VEGF), and insulin-like growth factor (IGF) [7]. Abundant evidence suggests that PDGF activates collagenase, stimulates fibronectin production, and is required for collagen remodeling in normal wound healing [8]. Moreover, some studies have indicated that the quantification of PDGF-BB released by PRP positively correlates with the healing rate [9].

By comparing the results of our second and third cases, we noted that the second case showed better collagen density improvement. The level of PDGF-BB in the second case was also higher than that in the third case. Therefore, we hypothesized that the quality of L-PRP (with PDGF-BB level as an indicator) is positively correlated with collagen remodeling. The level of PDGF-BB might predict the outcome of aesthetic treatment. More data are required to obtain the ideal concentration for neocollagenesis and facial rejuvenation.

For routine PRP preparation, patients have to have blood drawn each time before centrifugation due to the short shelf life of PRP [10]. For L-PRP preparation, $250 \mathrm{ml}$ of autologous blood was collected at once. L-PRP provides a longer storage period with measured PDGF-BB levels. It can be easily reconstituted before treatment sessions. In addition, lidocaine solution can be added to normal saline for reconstitution to improve injection comfort. After reconstitution, most of the platelets retained their original shapes (Fig. 1), which is different from the qualified human platelet lysate.

L-PRP can be considered an effective treatment to increase patients' healing rate with deep second-degree burn injury [6]. Intra-articular injection of freeze-dried platelet-derived factor concentrate for knee osteoarthritis effectively improved pain, sports, and recreational activities [2]. Our data demonstrated that lyophilized platelets could also be used for skin rejuvenation. The results of our study also suggested that lyophilized platelet power preserved platelet bioactivity. As the PDGF-BB level is measured, we can obtain different PDGF-BB concentrations by adding little reconstitution solutions rather than $1 \mathrm{ml}$. However, this study had certain limitations. We collected the samples in a few cases in this study. Therefore, more cases and data collection are required in further studies to identify the ideal growth factor levels, clinical outcomes, and neocollagenesis.

In conclusion, our results suggest that L-PRP can be used in facial esthetic treatment. Positive evidence was shown for increased collagen density in the dermis and subcutaneous layers. No obvious fibrotic changes or foreign body reactions were noted. In addition, the improvement noted by the instruments correlated with the improvement of skin texture, decreased wrinkles, and positive clinical outcomes. These results highlight L-PRP's potential as an efficient and convenient process compared to the original PRP in skin rejuvenation treatment.

\section{Conflicts of interest}

The author has nothing to disclose.

\section{References}

1. Tsay RC, Vo J, Burke A, Eisig SB, Lu HH, Landesberg R. Differential growth factor retention by platelet-rich plasma composites. J Oral Maxillofac Surg 2005;63:521-8.

2. Dhurat R, Sukesh M. Principles and methods of preparation of platelet-rich plasma: a review and author's perspective. J Cutan Aesthet Surg 2014;7:189-97.

3. Peng GL. Platelet-rich plasma for skin rejuvenation: facts, fiction, and pearls for practice. Facial Plast Surg Clin North Am 2019;27:405-11. 
4. Frautschi RS, Hashem AM, Halasa B, Cakmakoglu C, Zins JE. Current evidence for clinical efficacy of platelet rich plasma in aesthetic surgery: a systematic review. Aesthet Surg J 2017;37:353-62.

5. Mariani E, Pulsatelli L. Platelet concentrates in musculoskeletal medicine. Int J Mol Sci 2020;21:1328.

6. Shirata T, Kato Y. Can intra-articular injection of freeze-dried platelet-derived factor concentrate regenerate articular cartilage in the knee joint? Regen Ther 2019;11:5-7.

7. Pierce GF, Mustoe TA, Altrock BW, Deuel TF, Thomason A. Role of platelet-derived growth factor in wound healing. J Cell Biochem 1991;45:319-26.

8. Verma R, Negi G, Kandwal A, Chandra H, Gaur DS, Harsh M.
Effect of autologous PRP on wound healing in dental regenerative surgeries and its correlation with PDGF levels. Asian J Transfus Sci 2019;13:47-53.

9. da Silva LQ, Montalvão SAL, Justo-Junior ADS, Cunha Júnior JLR, Huber SC, Oliveira CC, et al. Platelet-rich plasma lyophilization enables growth factor preservation and functionality when compared with fresh platelet-rich plasma. Regen Med 2018;13:775-84.

10. Yeung CY, Hsieh PS, Wei LG, Hsia LC, Dai LG, Fu KY, et al. Efficacy of lyophilised platelet-rich plasma powder on healing rate in patients with deep second degree burn injury: a prospective double-blind randomized clinical trial. Ann Plast Surg 2018;80(2S Suppl 1):S66-9. 